\title{
Study of gastrointestinal stromal tumors with c-kit immunostaining
}

\author{
Sigdel $\mathrm{B}^{1}$, Vaidya $\mathrm{S}^{1}$, KC Sabira ${ }^{1}$ \\ ${ }^{1}$ Department of Pathology, Patan Academy of Health Sciences, School of Medicine, Nepal
}

\section{Keywords: \\ Gastrointestinal; Stromal tumors; C-KIT oncogene}

\begin{abstract}
Background: Mutation of the C-KIT oncogene is the central event in gastrointestinal stromal tumors which are the most common mesenchymal tumors arising from the tubular gastrointestinal tract.

Materials and Methods: A study was conducted in Patan hospital from April 2003 to May 2010. Mesenchymal tumors arising from the tubular gastrointestinal tract with the microscopic features suggesting gastrointestinal stromal tumor were included in the study.

Results: A total of 22 cases were studied. The incidence was highest amongst the older age group $(86.36 \%)$, than in younger people $(13.63 \%)$. The tumor most commonly involved the small intestine (54.54\%), followed by the stomach (36.36\%). Most (59.09\%) of the tumors were of huge size measuring $>100 \mathrm{~mm}$, and showed necrosis, hemorrhage and cystic degeneration. Mitotic activity was high (>5/50 high power fields) in $55 \%$ of the cases.
\end{abstract}

Conclusion: C-KIT immunostaining showed positivity in $19(86.36 \%)$ of the tumors in this study. Mutation of the C-KIT oncogene is seen in most of the gastrointestinal stromal tumors.

\section{INTRODUCTION}

Gastrointestinal stromal tumors (GISTs) are C-KIT (CD117)-positive, mesenchymal tumors of the gastrointestinal (GI) tract encompassing a majority of tumors previously considered GIST. ${ }^{1-2}$ They are believed to arise from the interstitial cells of Cajal or related stem cells ${ }^{1}$ primarily within the muscular wall of stomach (50-70\%) and small intestine $(20-30 \%){ }^{2}$ The rest are found in esophagus, large intestine and anus. In most cases of GISTs there is mutation of C-KIT oncogene, found in all cells of the body and responsible for formation of a protein called KIT. Mutually exclusive KIT and platelet derived growth factor receptor alpha (PDGFRA) mutations are central events in GIST pathogenesis. ${ }^{3}$ Ninety five percent of GISTs are CD-

\section{Correspondence:}

Dr. Bandana Sigdel, $M D$

Department of Pathology, Patan Academy of Health Sciences,

School of Medicine, Nepal

E-mail: bandana_sigdel@hotmail.com
117 positive. Immunohistochemical confirmation of the diagnosis using these antibodies and complete exclusion of other similar appearing tumors are extremely important. Histologically, most cases fall into one of these three categories: spindle cell type (70\%), epithelioid type $(20 \%)$ and mixed cell type (10\%). The objective was to study the incidence and distribution of age, sex, ethnicity, site of involvement as well as to asses the C-KIT immunostaining in GIST.

\section{MATERIALS AND METHODS}

This was retrospective study done over a period of 7 years and 2 months at Patan Academy of Health Sciences, which included 22 cases of GISTs arising from the GI tract. Hospital records were reviewed for clinical history. The samples received in the lab was grossed, processed, sectioned and stained with Haematoxylin and Eosin stains. These slides were reviewed by the pathologist. CD117 
immunostaining was performed by HRP POLYMER DETECTION TECHNIQUE. The cases were also analyzed for the incidence, age, sex, ethnicity, sites of involvement in GI tract, nature of the tumor considering tumor size and mitotic activity as defining criteria, and the secondary features.

\section{RESULTS}

There were 22 cases of GIST which represented $83 \%$ of the GI mesenchymal tumors and $3 \%$ of the total GI malignancies. $70 \%$ of the tumors were in the high risk category and $30 \%$ in the low risk category.

The tumor was more common among female patients with a male: female ratio of $1: 1.6$. Most of the tumors $(86 \%)$ were seen in patients above 40 years of age. The tumor most commonly involved the small intestine $(54.5 \%)$, followed by stomach $(36.4 \%)$ and the colorectum $(9.1 \%$, fig. 1$)$. None of the tumors arose from the esophagus. Out of the $54.5 \%$ of the small intestine tumors, $75 \%$ of the small intestinal tumors involved the ileum and the remaining $25 \%$ involved the Jejunum (fig. 2).
Microscopically the tumors were composed of spindled cells in fascicles and had plump to elongated nuclei and eosinophilic cytoplasm (fig. 3). Some of the tumors showed significant cellular atypia with frequent mitotic activity and necroses while the others were uniform with low to rare mitotic activity and absence of necrosis. Spillage of tumor into the surrounding tissue was seen in some of the large sized tumors with obvious features of malignancy.

The result of C-KIT Immunostaining is presented in Table 1, fig. 4.

Most (59.1\%) of the tumors were of huge size measuring $>100 \mathrm{~mm}, 18.2 \%$ were between $21-50 \mathrm{~mm}$, and $22.7 \%$ between $51-100 \mathrm{~mm}$. None of them were less than $20 \mathrm{~mm}$ in size. The large tumors showed presence of necrosis, hemorrhage and cystic degeneration. Necrosis was found in $70 \%$, hemorrhage in $72.7 \%$, and cystic degeneration in

Table 1: Result of C-KIT Immunostaining

\begin{tabular}{ccc}
\hline C-KIT Positive & C-KIT Negative & Total \\
\hline 19 cases & 3 cases & 22 \\
$(86.86 \%)$ & $(36.36 \%)$ & \\
\hline
\end{tabular}
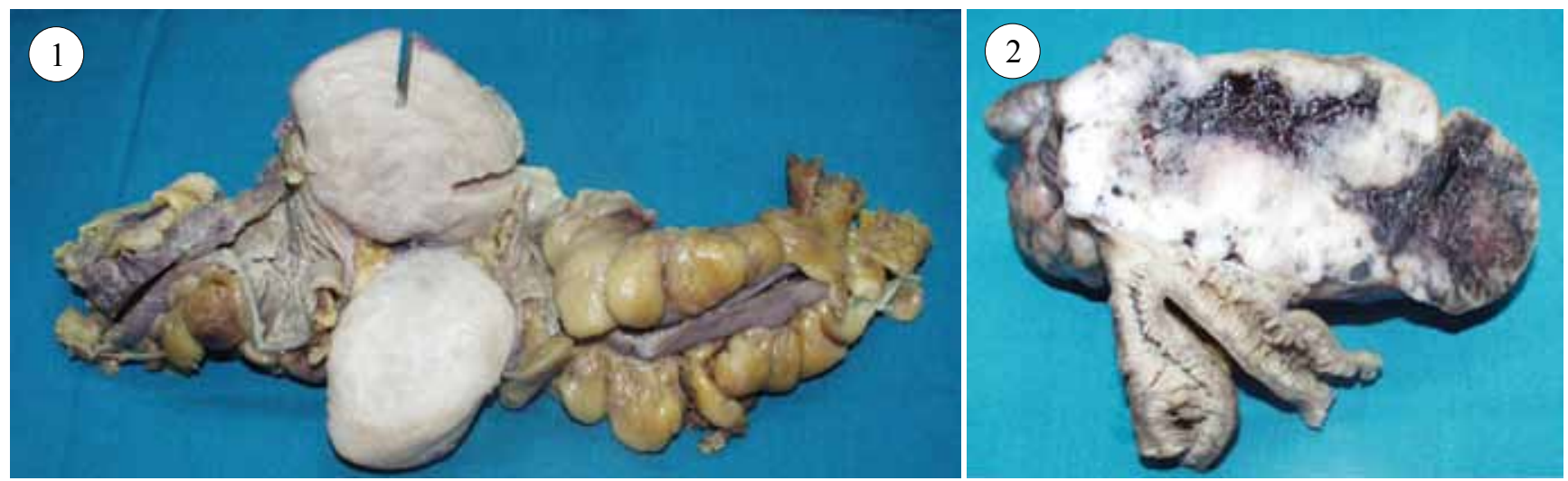

Figure 1: Gross photograph showing well circumscribed gray white tumor arising from colon.

Figure 2: Gross photograph showing tumor arising from jejunum with areas of necrosis.

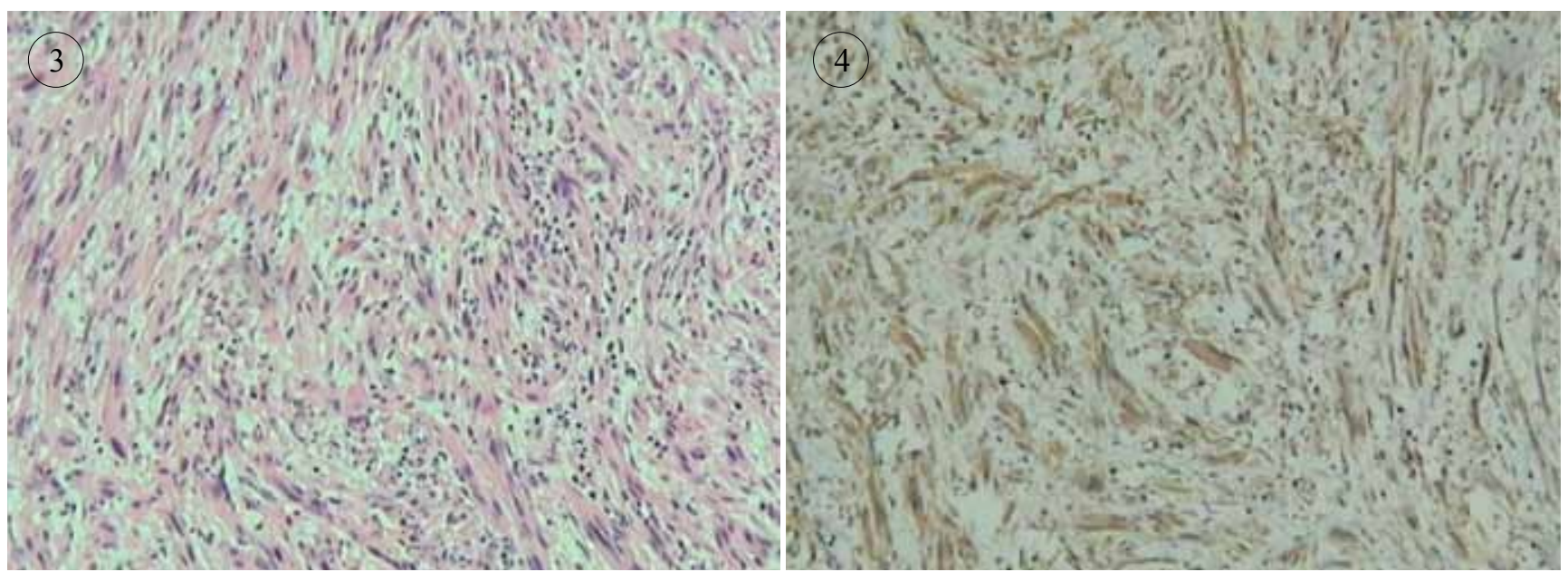

Figure 3: Photomicrograph composed of spindled cells in fascicles with plump to elongated nuclei and eosinophilic cytoplasm (HE, X10).

Figure 4: Photomicrograph showing tumor cells with diffuse C-KIT positivity (X10). 
$36.4 \%$ of the cases. Mitotic activity was high $(>5 / 50 \mathrm{hpfs})$ in $63.6 \%$ of the cases and low $(<5 / 50 \mathrm{hpfs})$ in $36.4 \%$ of the cases.

The tumor was most commonly found among the Mongolians 11 cases (50\%), followed by Newars 7 cases (31.8\%) and Brahmins/Chhetris 3 cases (13.6\%).

\section{DISCUSSION}

GISTs are rare, constituting less than 3\% of GI malignant neoplasias and rank a distal third in prevalence behind adenocarcinomas and lymphomas. Eighty percent of the mesenchymal tumors of the GI tract are GIST. ${ }^{2,}{ }^{4}$ Most tumors referred to as leiomyomas or leiomyosarcomas in the older literature are actually GIST. Only with tumors in the esophagus does the term leiomyoma remain accurate. In this study there were only 22 cases of GISTs which accounted for $2.5 \%$ of the malignant GI tumors. It represented $83 \%$ of the GI mesenchymal tumors and thus were the most common one. GISTs are rare in the esophagus and are more common in stomach and small bowel. They represent $20 \%$ of small bowel malignant neoplasms, $1-2 \%$ of gastric malignancies and less than $1 \%$ of malignancies involving the esophagus, colon and rectum.

In this study majority of the tumors were found to arise from the small intestine (54.54\%) and stomach (36.36\%) and none involved the esophagus. Colorectum $(9.09 \%)$ was much less commonly involved. In the small intestine, ileum (75\%) was a much more common site than jejunum (25\%). Duodenal involvement was not seen. GISTs of appendix are rare $^{5}$ and it was not seen in our study. Miettinen and Sobin, ${ }^{6}$ however, described 4 cases of GISTs of appendix in adult males.

Most of the studies have shown the incidence of GIST to be equal in male and female, while a few have shown slight male preponderance. In this study it showed a higher prevalence among females with a ratio of $1: 1.75$. This may be due to the small sample size in the study. GISTs usually occur in the older adults and rarely in children in the second decade. ${ }^{1}$ Most studies have also found the tumor common in older age group with the peak in $5^{\text {th }}$ and $6^{\text {th }}$ decades.

Similarly, we found most of the tumors in older people $(86 \%)$ and only a single case was found in patient less than 20 years. The youngest patient was 19 years and the oldest 76 years.

Tumor size, mitotic rate and location can be used to predict the risk of recurrence in GIST patients. Tumors $<2 \mathrm{~cm}$ with a mitotic rate of $<5 / 50 \mathrm{HPF}$ have been shown to have lower risk of recurrence than the larger or more aggressive tumors. Nevertheless, all GIST tumors should be considered to have malignant potential and no GIST can be correctly classified as benign. ${ }^{7}$ More than $50 \%$ of the cases in the study were
$>100 \mathrm{~mm}$ in size along with high mitotic activity, indicating their aggressive nature. Mitotic rate was higher in large sized tumors with presence of secondary features. 59.1\% of the cases were circumscribed and $40.9 \%$ showed tumor spillage. Overall $70 \%$ of the cases were in high risk category and $30 \%$ in the low risk category.

Most GISTs show strong diffuse cytoplasmic staining for CD $117(90 \%)$ of cells and up to 50\% show clot like pattern. A few show focal staining ( 5 to $50 \%$ of cells). Positivity for KIT tyrosine kinase receptor (stem cell receptor factor) is perhaps their single best defining factor. ${ }^{8}$ In our study 19 cases $(86.86 \%)$ were positive for C-KIT, thus confirming the morphological impression. The remaining three cases were probably those lacking kit mutations. These may have intragenic activation mutations in the related receptor tyrosine kinase, platelet derived growth factor receptor alpha (PDGFRA) and could have stained positively for it. The KIT positive patients have been receiving imatinib and majority are showing good response.

The tumors were most commonly found among the Mongolians 11 cases (50\%), followed by Newars 7 cases (31.8\%) and Brahmins/Chhetris 3 cases (13.6\%). The variation in the rate of occurrence of disease among different ethnic groups may be related to genetic susceptibility for the disease.

There is increased incidence of GISTs including multiple small intestinal tumors in patients with von Recklinghausen's neorofibromatosis (NF-1) and is possibly associated with interaction between the NF-1 gene product and C-KIT. ${ }^{9}$ Non of the patients in this study had such association.

\section{CONCLUSION}

This study shows that GIST, a distinct type of neoplasm, arising from the specialized cells within the muscular layer of GI tract. C-KIT immunostaining is important and should be routinely practiced along with other markers like PDGFRA and CD34.

\section{REFERENCES}

1. Miettinen M, Lasota J. Gastrointestinal stromal tumors: review on morphology, molecular pathology, prognosis, and differential diagnosis. Arch Pathol Lab Med 2006;130:1466-78.

2. Lasota J, Miettinen M. Clinical significance of oncogenic KIT and PDGFRA mutations in gastrointestinal stromal tumors. Histopathology 2008;53:245-66.

3. Lasota J, Miettinen M. KIT and PDGFRA mutations in gastrointestinal stromal tumors (GISTSs). Semin Diagn Pathol 2006;23:91-102.

4. Corless CL, Schroeder A, Griffith D et al. PDGFRA mutations in gastrointestinal stromal tumors: frequency, spectrum and in vitro sensitivity to imatinib. J Clin Oncol 2005;23:5357-64.

5. Fisher C. Recent Advances in Histopathology In: Gastrointestinal Stromal Tumors: The Royal Society of Medicine Press Limited; 2003. pp 71-78 
6. Miettinen M, Sobin LH. Gastrointestinal stromal tumors in the appendix: a clinicopathological and immunohistochemical study of four cases. Am J Surg Pathol 2001;25:1433-7.

7. Raut CP, DeMatteo, RP. Evidence-guided surgical management of GIST: beyond a simple case of benign and malignant. Ann Surg Onc 2008; $15: 426$
8. Miettinen M, Blay JY, Sobin LH. WHO Classification of tumors pathology and genetics of tumors of the digestive system. IARC Press: Lyon; 2000. pp 62-65.

9. Kinoshita K, Hirota S, Isozaki K et al. Absence of C-KIT mutation in gastrointestinal stromal tumors from neurofibromatosis type 1 patients. J Pathol 2004;202:80-5. 